\title{
Maximum and minimum problems in secondary school education
}

\author{
ZSOLT FÜLÖP
}

Abstract. The aim of this paper is to offer some possible ways of solving extreme value problems by elementary methods with which the generally available method of differential calculus can be avoided. We line up some problems which can be solved by the usage of these elementary methods in secondary school education. The importance of the extremum problems is ignored in the regular curriculum; however they are in the main stream of competition problems - therefore they are useful tools in the selection and development of talented students. The extremum problem-solving by elementary methods means the replacement of the methods of differential calculus (which are quite stereotyped) by the elementary methods collected from different fields of Mathematics, such as elementary inequalities between geometric, arithmetic and square means, the codomain of the quadratic and trigonometric functions, etc. In the first part we show some patterns that students can imitate in solving similar problems. These patterns could also provide some ideas for Hungarian teachers on how to introduce this topic in their practice. In the second part we discuss the results of a survey carried out in two secondary schools and we formulate our conclusion concerning the improvement of students' performance in solving these kind of problems.

Key words and phrases: maximum and minimum problems, theorem of the means, elementary methods, learning difficulties, functions of several variables, the method of the indetermined parameters.

ZDM Subject Classification: D20, D50, D70, I10, I40.

\section{Introduction}

In the secondary school education the extreme value problems, or maximum and minimum problems, or problems concerned with the greatest and the least values, are more attractive than other mathematical problems. We may observe 
that everyday life problems are very often maximum and minimum problems of a sort. We wish to obtain a certain object at the lowest price, or the greatest possible effect with a certain effort, or a certain effect with the minimum cost, or the maximum work done within a given time. The physicists gave clear and useful forms to ideas of this sort; they describe certain physical phenomena in terms of "minimum principles". The first dynamical principle of this kind, the "Principle of Least Action" (which usually goes under the name of Maupertius), was essentially developed by Euler.

The differential calculus provides a general method for solving problems on minima and maxima. The present day secondary school curriculum does not contain the elements of differencial and integral calculus (we do not mean the secondary schools with advanced mathematical teaching programme). But it does not mean that we cannot deal with extreme value problems in secondary school educational processes, since the most of these problems can be solved by elementary methods. Therewith, we can solve, by elementary methods, many problems which need the partial differential of a function of several real variables (and this method is not included in any secondary school curriculum). In short, the problem-solving by elementary methods means the replacement of the methods based on differential calculus, which are quite stereotyped, by the elementary methods collected from different fields of Mathematics, such as elementary inequalities between geometric, arithmetic and square means, the codomain of the quadratic and trigonometric functions, etc. In the case of elementary methods there are no generally valid rules to solve the problems, every exercise is a particular problem. However, having solved a problem with real insight and interest, the students aquire a precious possession: a pattern, a model, that they can imitate in solving similar problems. They develop this pattern if they try to follow it, if they reflect upon the analogy of problems solved, upon the relevant circumstances that make a problem accessible to this kind of solution, etc. Developing such a pattern, they may finally attain a real discovery and they have a chance to aquire some well ordered and readily available knowledge. In this way, we consider a restricted usage of the differencial calculus is legitimate, however we do not undervalue the enormous scientific importance of the differencial and integral calculus. These chapters of the mathematical analysis are very useful, because they make it possible to handle a lot of problems which cannot be solved by elementary methods. While we teach the mathematical analysis (of course, in the schools with advanced mathematical teaching programme) we have to underline this kind of problems in order to point out the value of the acquired knowledge, 


$$
\text { "05-fulop" — 2015/5/22 — 0:49 — page } 83 \text { — \#3 }
$$

but we have to avoid the discussion of problems which can also be solved by elementary methods.

\section{Possible applications in secondary school}

Solving extreme value problems is a challenge for secondary school students. The Hungarian mathematics curriculum includes this type of problems for 10th grade students (see [7]). Most of the problems are in conjunction with the maximum or minimum points of a quadratic function, and the basic inequality between geometric, arithmetic and square means. In the following we would like to offer an other point of view concerning this type of problems, and to show some new patterns that the Hungarian teachers could use in teaching of (not only) talented students.

\subsection{The theorem of the arithmetic and geometric means and its} consequences

In the secondary school educational processes we can use the following theorems.

THEOREM 1. The product of $n$ positive real numbers with a given sum becomes a maximum when these numbers are all equal.

TheOREM 2. The sum of $n$ positive real numbers with a given product becomes a minimum when these numbers are all equal.

Theorem 1 and 2 are different formulations of the so-called theorem of the arithmetic and geometric means or, shortly, the theorem of the means. This theorem states that the arithmetic mean of a list of non-negative real numbers is greater than or equal to the geometric mean of the same list; and further, that the two means are equal if and only if every number of the list is the same. Namely

$$
\frac{x_{1}+x_{2}+\cdots+x_{n}}{n} \geq \sqrt[n]{x_{1} \cdot x_{2} \cdots x_{n}}
$$

and the equality holds if and only if $x_{1}=x_{2}=\cdots=x_{n}$.

It is simply, however, to observe that the inequality between the means yields both of the theorems: to obtain one or the other, we have to regard one or the other side of the inequality (1) as given. The simplest non-trivial case (i.e. with 


$$
\text { "05-fulop" — 2015/5/22 — 0:49 — page 84 — \#4 }
$$

more than one variable) for two non-negative numbers $x_{1}$ and $x_{2}$, is the statement that

with equality if and only if $x_{1}=x_{2}$.

$$
\frac{x_{1}+x_{2}}{2} \geq \sqrt{x_{1} \cdot x_{2}}
$$

We can see that Theorem 1 is concerned with a maximum, Theorem 2 with the corresponding minimum. We may call these two statements conjugate statements.

If we regard one or the other side of the inequality (2) as given then we get the following theorems.

THEOREM 3. The product of two positive real numbers with a given sum becomes a minimum when these numbers are equal.

THEOREM 4. The sum of two positive real numbers with a given product becomes a minimum when these numbers are equal.

We have to mention that Pólya gave an interesting geometrical interpretation to prove Theorem 3 (see [6]).

THEOREM 5. The product $p=x_{1}^{m} \cdot x_{2}^{n}$, where $x_{1}$ and $x_{2}$ are two positive real numbers with a given sum and $m, n \in \mathbb{N}$, becomes a maximum when $\frac{x_{1}}{m}=\frac{x_{2}}{n}$.

ProOF. The product $p=x_{1}^{m} \cdot x_{2}^{n}=m^{m} \cdot n^{n} \cdot \frac{x_{1}^{m}}{m^{m}} \cdot \frac{x_{2}^{n}}{n^{n}}$ becomes a maximum, when the product $\frac{x_{1}^{m}}{m^{m}} \cdot \frac{x_{2}^{n}}{n^{n}}$ becomes a maximum, too. We can see that

$$
\frac{x_{1}^{m}}{m^{m}} \cdot \frac{x_{2}^{n}}{n^{n}}=\overbrace{\frac{x_{1}}{m} \cdot \frac{x_{1}}{m} \cdots \frac{x_{1}}{m}}^{m \text { times }} \cdot \overbrace{\frac{x_{2}}{n} \cdot \frac{x_{2}}{n} \cdots \frac{x_{2}}{n}}^{n \text { times }}
$$

and

$$
\overbrace{\frac{x_{1}}{m}+\frac{x_{1}}{m}+\cdots+\frac{x_{1}}{m}}^{\text {times }}+\overbrace{\frac{x_{2}}{n}+\frac{x_{2}}{n}+\cdots+\frac{x_{2}}{n}}^{n \text { times }}=m \cdot \frac{x_{1}}{m}+n \cdot \frac{x_{2}}{n}=x_{1}+x_{2}=s
$$

is the given sum, therefore the product $p$ becomes a maximum when $\frac{x_{1}}{m}=\frac{x_{2}}{n}$, by virtue of Theorem 1 .

We formulate, without any proof, the conjugate statement of Theorem 5 :

TheOREM 6. If the product $p=x_{1}^{m} \cdot x_{2}^{n}$ is given, where $x_{1}$ and $x_{2}$ are two positive real numbers and $m, n \in \mathbb{N}$, then the sum $s=x_{1}+x_{2}$ becomes a minimum when $\frac{x_{1}}{m}=\frac{x_{2}}{n}$. 


$$
\text { "05-fulop" — 2015/5/22 — 0:49 — page 85 — \#5 }
$$

We can generalize Theorem 5 as follows:

THEOREM 7. The product $p=x_{1}^{\alpha_{1}} \cdot x_{2}^{\alpha_{2}} \cdots x_{n}^{\alpha_{n}}$, where $x_{1}, x_{2}, \cdots, x_{n}$ are $n$ positive real numbers with a given sum and $\alpha_{1}, \alpha_{2}, \cdots, \alpha_{n} \in \mathbb{Q}$, becomes a maximum when $\frac{x_{1}}{\alpha_{1}}=\frac{x_{2}}{\alpha_{2}}=\cdots=\frac{x_{n}}{\alpha_{n}}$.

The proof of the foregoing theorem can be found in [2], so we omit its detailed presentation.

The conjugate statement of the Theorem 7 is the following:

TheOREM 8. If the product $p=x_{1}^{\alpha_{1}} \cdot x_{2}^{\alpha_{2}} \cdots x_{n}^{\alpha_{n}}$ is given, where $x_{1}, x_{2}, \cdots, x_{n}$ are $n$ positive real numbers and $\alpha_{1}, \alpha_{2}, \cdots, \alpha_{n} \in \mathbb{Q}$ then the sum $s=x_{1}+x_{2}+$ $\cdots+x_{n}$ becomes a minimum when $\frac{x_{1}}{\alpha_{1}}=\frac{x_{2}}{\alpha_{2}}=\cdots=\frac{x_{n}}{\alpha_{n}}$.

\subsection{Patterns and examples}

Problem 1. Being given $b$, the length of the leg of an isosceles triangle, find the maximum of its area!

Pattern 1: We denote the half of the base by $x$, the altitude of the isosceles triangle by $h$, and, by virtue of the Pythagorean theorem,

$$
h=\sqrt{b^{2}-x^{2}}
$$

follows. The area of the triangle is

$$
A=x \cdot \sqrt{b^{2}-x^{2}}=\sqrt{x^{2} \cdot\left(b^{2}-x^{2}\right)} .
$$

The area becomes a maximum when the product $x^{2} \cdot\left(b^{2}-x^{2}\right)$ is a maximum. We consider the quadratic function $f(x)=y \cdot\left(b^{2}-y\right)$, where $y=x^{2}$, which becomes a maximum when $x=b \cdot \frac{\sqrt{2}}{2}$. From (4) follows that the maximum value of the area is $A_{\max }=\frac{b^{2}}{2}$.

We can solve the problem almost in the same way if we choose the altitude of the triangle as a variable element.

We have to mention that we also can get equality (4) by the use of the Heron's formula.

Pattern 2: The sum $x^{2}+\left(b^{2}-x^{2}\right)=b^{2}$ is given, therefore we can apply Theorem 3 . The product $x^{2} \cdot\left(b^{2}-x^{2}\right)$ becomes a maximum when $x^{2}=b^{2}-x^{2}$ so $x=b \cdot \frac{\sqrt{2}}{2}$ follows. 


$$
\text { "05-fulop" — 2015/5/22 — 0:49 — page } 86 \text { — \#6 }
$$

Pattern 3: We consider the equalities $x=b \cdot \cos \alpha$ and $h=b \cdot \sin \alpha$ (where $\alpha$ is the angle between the base and the leg) therefore the area of the triangle is

$$
A=b^{2} \cdot \sin \alpha \cdot \cos \alpha=\frac{b^{2} \cdot \sin (2 \cdot \alpha)}{2} .
$$

The area becomes a maximum, when the value of $\sin (2 \cdot \alpha)$ is a maximum. Therefore $\alpha=\frac{\pi}{4}$ and $x=b \cdot \frac{\sqrt{2}}{2}$ follows.

Pattern 4: The area of the triangle is equal to $A=\frac{b^{2} \cdot \sin \gamma}{2}$, where $\gamma$ is the angle between legs. If $\gamma=\frac{\pi}{2}$ then $\sin \gamma=1$ and the area of the triangle becomes a maximum. The maximum value is $A_{\max }=\frac{b^{2}}{2}$.

Pattern 5: The isosceles triangle can be considered as a half of a rhombus with given side $b$. We know that of all rhombi with a given side the square has the maximum area, so the maximum of the area of the triangle is $A_{\max }=\frac{b^{2}}{2}$.

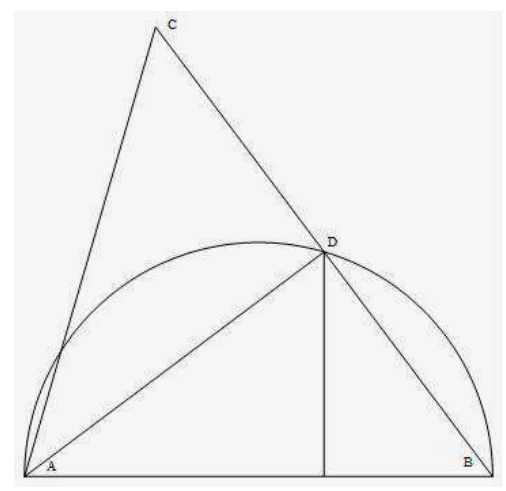

Figure 1

Pattern 6: The area of the $\triangle A B C$ triangle (where $A B=A C=b$ ) becomes a maximum when the area of the $\triangle A B D$ triangle (the half of the $\triangle A B C$ triangle) becomes a maximum. $\triangle A B D$ is a right angled triangle so we can draw a circle on $A B$ as diameter and $D$ is a point of this circle by virtue of Thales' theorem. The area of the $\triangle A B D$ triangle becomes a maximum when the altitude to the given hypotenuse becomes a maximum and it happens in the case of an isosceles right triangle (see Figure 1). Therefore $B D=A D=$ $b \cdot \frac{\sqrt{2}}{2}$.

In several problems we have to seek the extremum (minimum or maximum) of a quantity depending on two or more variables. The simultaneous variation of all 


$$
\text { "05-fulop" — 2015/5/22 — 0:49 — page } 87-\# 7
$$

variables, or total variation, is not easy to survey. To solve this kind of problem we fix for a moment all originally variable elements except one and study the effect of the variation of this single element. The theoretical principle underlying this pattern is: a function of several variables cannot attain a maximum (or minimum) with respect to all its variables jointly, unless it attains a maximum (or minimum) with respect to each single variable. As an example we introduce the following problem.

Problem 2. In an isosceles trapezium the sum of the lengths of three sides (two legs and a base) is given. Find the maximum of its area!

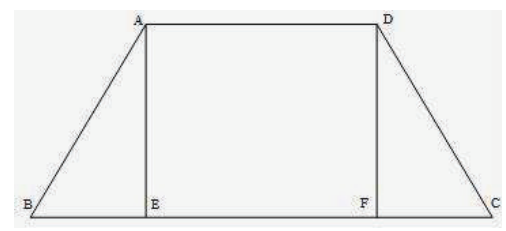

Figure 2

Pattern 1: We denote the given sum by $s$. Let be the first variable element the length of a leg, denoted by $x$, and the second variable element the angle between the leg and the bigger base, denoted by $\alpha=\angle A B E$ (see Figure 2). Therefore the length of the smaller base is equal to $s-2 \cdot x$.

The area of the trapezium is

$$
A=s \cdot x \cdot \sin \alpha-x^{2} \cdot \sin \alpha \cdot(2-\cos \alpha)
$$

In the first step we fix the variable element $\alpha$ and study the effect of the variation of the element $x$. Since $\alpha \in] 0, \frac{\pi}{2}[$ the area becomes a maximum when

$$
x=\frac{s}{2 \cdot(2-\cos \alpha)},
$$

and the maximum value of the area is

$$
A_{\max }(\alpha)=\frac{s^{2}}{4} \cdot \frac{\sin \alpha}{2-\cos \alpha},
$$

but, obviously, this maximum value depends on $\alpha$. Since $\frac{s^{2}}{4}$ is given, in order to maximalize the area we have to find the value of $\alpha$ such that the fraction 


$$
\text { "05-fulop" — 2015/5/22 — 0:49 — page 88 — \#8 }
$$

$\frac{\sin \alpha}{2-\cos \alpha}$ becomes a maximum. We make the substitution $\tan \frac{\alpha}{2}=u$ and we have

$$
\frac{\sin \alpha}{2-\cos \alpha}=\frac{2 \cdot u}{1+3 \cdot u^{2}}
$$

The fraction $\frac{2 \cdot u}{1+3 \cdot u^{2}}$ becomes a maximum when its reciprocal

$$
\frac{1+3 \cdot u^{2}}{2 \cdot u}=\frac{1}{2 \cdot u}+\frac{3 \cdot u}{2}
$$

becomes a minimum. But the product $\frac{1}{2 \cdot u} \cdot \frac{3 \cdot u}{2}=\frac{3}{4}$ is given, so the sum $\frac{1}{2 \cdot u}+\frac{3 \cdot u}{2}$ becomes a minimum when $\frac{1}{2 \cdot u}=\frac{3 \cdot u}{2}$, by virtue of Theorem 2 , therefore $u=\frac{1}{\sqrt{3}}$ and $\alpha=\frac{\pi}{3}$ follows. The maximum value of the area is

$$
A_{\max }=\frac{a^{2} \cdot \sqrt{3}}{12} .
$$

Pattern 2: Let us try an other way to choose the variable elements. The first variable remain the length of a leg, denoted by $x$, and the second variable element became the length $B E=z$ (see Figure 2).

The area of the trapezium is

$$
A=(s-2 \cdot x+z) \cdot \sqrt{x^{2}-z^{2}} .
$$

We can see that the area becomes a maximum when the product $(s-2 \cdot x+$ $z)^{2} \cdot(x-z) \cdot(x+z)$ attains its maximum. Theorem 7 seems to be an adequate method to solve this problem. The main difficulty is there are no $x$ nor $z$ values such that $s-2 \cdot x+z, x-z$ and $x+z$ are pairwise equal. So we have to introduce two parameters $m$ and $n$ such that $\frac{s-2 \cdot x+z}{2}=m \cdot(x-z)=$ $n \cdot(x+z)$. We have to determine the values of the parameters $m$ and $n$ such that the sum

$(s-2 \cdot x+z)+(m \cdot x-m \cdot z)+(n \cdot x+n \cdot z)=s+(m+n-2) \cdot x+(1-m+n) \cdot z$

be given (it depends neither on $x$ nor on $z$ ), according to the requirements of Theorem 7. Therefore we have

$$
m+n-2=0 \quad 1-m+n=0
$$

and the values $m=\frac{3}{2}$ and $n=\frac{1}{2}$ follow.

By virtue of Theorem 7 , the product $(s-2 \cdot x+z)^{2} \cdot \frac{3 \cdot x-3 \cdot z}{2} \cdot \frac{x+z}{2}$ becomes a maximum when $\frac{s-2 \cdot x+z}{2}=\frac{3 \cdot x-3 \cdot z}{2}=\frac{x+z}{2}$. Therefore 


$$
\text { "05-fulop" — 2015/5/22 — 0:49 — page } 89 \text { — \#9 }
$$

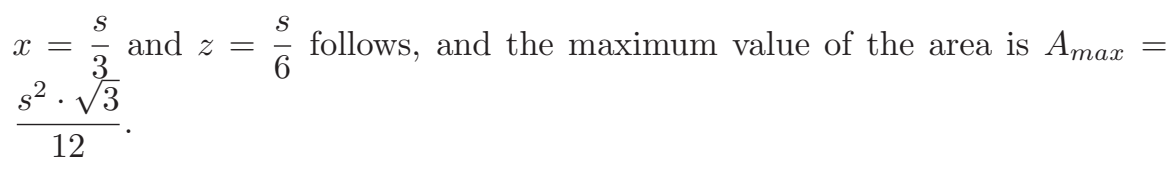

REMARK. We may call the foregoing pattern the method of the indetermined parameters, as we refer to the parameters $m$ and $n$, whose values, at first, were not known.

\section{Testing and results}

This study was carried out with a group of 79 secondary school students in grades 10 and 11. The two schools selected were Boronkai György Technical High School (Vác town, Hungary) and Reformed High School from Gödöllö. Both of the schools gather a part of the elite pupils in their region. The students have been specially selected for this study by their teachers. All of these students have a serious attitude towards the study of Mathematics, however they know nothing about the differential and integral calculus. Our first task was to survey how the students can handle extreme-value problems by elementary methods and which are the largest deficiencies and shortcomings in their problem solving skills concerning the extreme value problems. Every student received a test-paper with four exercises on it, from which they had to solve only three exercises, every student could omit an exercise (our intention also was to investigate which are the most agreeable exercises). The exercises were chosen by the author, and the students have 60 minutes to solve them. The students were asked to write in detail their attempts even though they could not solve the problem entirely. The result of the test is the following.

Exercise 1 (grade 10): Being given $c=10$, the length of the hypotenuse of a right-angled triangle, find the maximum of its area!

Exercise 1 (grade 11): Being given $b=4$, the length of the leg of an isosceles triangle, find the maximum of its area!

Table 1 shows that the grade 10 students were more successful than the grade 11 ones, but we also have to mention that their exercise was easier than the grade 11 students' exercise. Several grade 10 students solved the exercise by the use of the inequalities between geometric, arithmetic and square means. 2 students used the inequality between geometric and square means: $\sqrt{a \cdot b} \leq \sqrt{\frac{a^{2}+b^{2}}{2}} \Rightarrow$ $a \cdot b \leq 50$ and the equality holds if and only if $a=b=\sqrt{50}$ (where $a$ and $b$ denotes 
Table 1

\begin{tabular}{|c|c|c|}
\hline & 10th grade & 11th grade \\
\hline Right answer & 34 & 10 \\
\hline Wrong answer & 9 & 20 \\
\hline No response & 3 & 3 \\
\hline
\end{tabular}

the catheti of the triangle). 3 students solved the problem in the following way: $\sqrt{a \cdot b} \leq \sqrt{\frac{a^{2}+b^{2}}{2}} \Rightarrow a \cdot b \leq 50$, so the maximum value of the product $a \cdot b$ is 50 , we solve the system of equations $a \cdot b=50$ and $a^{2}+b^{2}=100$, therefore $a=b=\sqrt{50}$ follows. 19 students gave the right answer without any detailed explication: the area $A=\frac{a \cdot b}{2}$ becomes a maximum when $a=b$ and the equality $a=b=\sqrt{50}$ follows from the phytagorean theorem $a^{2}+b^{2}=100$. Two of them gave a weak explication that the area of a rectangle with given parameter becomes a maximum when the rectangle is a square and our triangle is a half of a square. One student argued that all the maximum and minimum problems like this have the solution $a=b .4$ students used the inequality between geometric and arithmetic means, one of them wrote $\frac{a+b}{2} \geq \sqrt{a \cdot b} \Rightarrow \frac{a^{2}+2 \cdot a \cdot b+b^{2}}{4} \geq a \cdot b \Rightarrow$ $25 \geq \frac{1}{2} \cdot a \cdot b$ and the product $a \cdot b$ becomes a maximum when $a=b=\sqrt{50}$ . One student used the right triangle altitude theorem and his solution is the following: $A=\frac{c \cdot \sqrt{p \cdot q}}{2}=5 \cdot \sqrt{p \cdot q}$ (where $p$ and $q$ are the two segments of the hypotenuse) and $\sqrt{p \cdot q} \leq \frac{p+q}{2}=5$, the equality $\sqrt{p \cdot q}=5$ holds if and only if $p=q=5$, so $a=b=\sqrt{50}$. 5 students drew the Thales circle on the hypotenuse as diameter, and they found that the area becomes a maximum when the altitude to the hypotenuse is equal to the radius of the circle. In the following we detail some wrong answers. 3 students gave the solution: $a^{2}+b^{2}=100 \Rightarrow a+b=10 \Rightarrow$ $\sqrt{a \cdot b} \leq \frac{a+b}{2}=5$ and the equality holds if and only if $a=b=5$. Two students thought that $A=\frac{a \cdot m_{a}}{2}$ (where $m_{a}$ denotes the altitude to the cathetus $a$ ) and the area becomes a maximum when the cathetus $a$ is as big as possible, one of the students gave the answer $a=9,99 \cdots$. One student wrote: $a+b>10$ (by virtue of the triangle inequality), $a=x+9, b=x$ and $a=19, b=10$ (he did not even realise that the result refers to an isosceles triangle and not to a right angled one). The grade 11 students had to solve Problem 1 from Section 2.2. We expected the solutions shown in Patterns 1-6. 5 students were successful applying Pattern 4. One student used Pattern 5 successfully. One student stated "the triangle must be a right angled one if the area is a maximum", without any argumentation, and then he gave the right answer. Another student's reasoning "Of all isosceles 
triangles the right angled one has the maximum area" and she also gave the right answer. One student argued in the following way: if $h=\frac{a}{2}$ then $h=2 \cdot \sqrt{2}$ and $A=8$; if $h>\frac{a}{2}$, for example $h=2 \cdot \sqrt{3}$ and $\frac{a}{2}=2$, then $A=4 \cdot \sqrt{3}<8$; if $h<\frac{a}{2}$, for example $h=\sqrt{5}$ and $\frac{a}{2}=3$, then $A=3 \cdot \sqrt{5}$ (here is a calculation mistake); and suddenly he turned to the inequality $\frac{\frac{a}{2}+h}{2} \geq \sqrt{\frac{a}{2} \cdot h}$ and he wrote that the equality holds if $h=\frac{a}{2}$, so $h=2 \cdot \sqrt{2}$ and $a=4 \cdot \sqrt{2}$, and the right answer follows (the base of the triangle is denoted by $a$, the altitude to the base is denoted by $h$, our unified notations). A complicated, but interesting answer is the following (we show a few sequences of it): " $A=\sqrt{16 \cdot h^{2}-h^{4}} ;-y^{2}+16 \cdot y-A^{2}=0$ (where $\left.y=h^{2}\right) ; y_{1 ; 2}=\frac{-16 \pm \sqrt{256-4 \cdot A^{2}}}{-2} ; y$ is a minimum when $256-4 \cdot A^{2}=0$ " (the student did not explain why we have to find the minimum of the $y$ ) " $\Rightarrow A=8$ and $y=8$, so $h=2 \cdot \sqrt{2}$ ". We have to mention that the correct argumentation is the following: $y$ is a real number so $256-4 \cdot A^{2} \geq 0$, therefore $A \in[-8,8]$ and the maximum value of the area is $A_{\max }=8$. A lot of students wrote the Pythagorean theorem $h^{2}+\frac{a^{2}}{4}=16$ and then they failed. 4 students thought the area becomes a maximum when the triangle is equilateral and made the calculation in this way. 3 students wrote that the base of the triangle is less than 8 , by virtue of the triangle inequality, so it is $a=7, \dot{9} .2$ students considered that the length of the base must be equal to the length of the altitude if the area is a maximum, one of them proceeded in the following way: $\frac{a^{2}}{4}+a^{2}=16 \Rightarrow a=\sqrt{\frac{64}{5}} \Rightarrow A_{\max }=\frac{32}{5}$.

Exercise 2 (grade 10): Find the maximum and minimum of the function $f$ : $[3,7] \rightarrow \mathbb{R}, f(x)=\sqrt{x-3}+\sqrt{7-x}$ !

Exercise 2 (grade 11): Find the maximum of the function $f:[-3,2] \rightarrow \mathbb{R}$, $f(x)=\sqrt{x+3}+2 \cdot \sqrt{2-x}$ !

We expected the grade 10 students will make the transformation $f^{2}(x)=4+$ $2 \cdot \sqrt{4-(x-5)^{2}}$ and, from the graph of the function $g(x)=-(x-5)^{2}+4$ on domain $x \in[3,7]$, the extreme values of the function $f$ follows. As Table 2 shows, most of the students (27) skip over this exercise. One student could solve the exercise in the way which we expected.

Table 2

\begin{tabular}{|c|c|c|}
\hline & 10th grade & 11th grade \\
\hline Right answer & 8 & 6 \\
\hline Wrong answer & 11 & 17 \\
\hline No response & 27 & 10 \\
\hline
\end{tabular}


Another student tried to apply this method but the result was the following: $f^{2}(x)=4+2 \cdot \sqrt{-x^{2}+10 \cdot x-21}$; (squaring once again) $f^{4}(x)-8 \cdot f^{2}(x)+16=$ $-4 \cdot x^{2}+40 \cdot x-84$ and the student did not know how to continue. 3 students plotted the graph of the functions $x \rightarrow \sqrt{x-3}$ and $x \rightarrow \sqrt{7-x}$ in the same coordinate system and they gave the right answer (they found the minimum and maximum points with the help of the graph and thereafter they calculated the corresponding minimum and maximum values). 4 students solved the equation $\sqrt{x-3}=\sqrt{7-x}$, thereafter they considered the maximum point is $x=5$ (the solution of the equation) and the corresponding maximum value is $f(5)=2 \cdot \sqrt{2}$, one of them gave the right answer concerning the minimum points of the function without any argumentation. We can say that these students found the right answer somehow accidentally. Another student gave the right answer accidentally, too, when he tried to use the inequality between arithmetic and geometric means and his argumentation is the following: the function $f$ becomes a maximum when the equality $\frac{\sqrt{x-3}+\sqrt{7-x}}{2}=\sqrt[4]{(x-3) \cdot(7-x)}$ holds and (he solved the equation) $x=5$ (and then he calculated the maximum value of the function). This student's argumentation concerning the minimum of the function was the following: to find the minimum we use the inequality $\frac{a^{2}+b^{2}}{4} \geq \frac{a+b}{2}$ and the substitutions $a=\sqrt{x-3}$ and $b=\sqrt{7-x}$ leads us to the inequality $1 \geq \frac{\sqrt{x-3}+\sqrt{7-x}}{2}$; the function becomes a minimum when the equality $1=\frac{\sqrt{x-3}+\sqrt{7-x}}{2}$ holds (a misinterpretation of the inequality between arithmetic and square means) and we get $x=3$ and $x=7$ (thereafter he calculated the corresponding minimum values). If we trace this train of thought carefully we can conclude that this student has some knowledge about the inequality between arithmetic, geometric and square means, but he does not know how to use properly this knowledge. 8 students wrote that the minimum and maximum value of the function are 3 and 7 respectively (they took the extreme values of the domain for the extreme values of the function).

Grade 11 students had no previous knowledge on how to solve Exercise 2. Our target was to survey how they can handle this kind of problem. We expected that most of the students will omit this exercise, but this did not happen, as Table 2 shows. 6 students tried to calculate the values of function in several points of the domain and 4 of them gave the right answer (in the case of 2 students the set of values did not contain $f(-2)$ so their answers were wrong). One student's work is the following (the others worked almost in the same way): "the domain of the function is $x \in[-3,2], f(-3)=2 \cdot \sqrt{5} ; f(-2)=5 ; f(-1)=\sqrt{2}+2 \cdot \sqrt{3}$; $f(0)=2 \cdot \sqrt{2}+\sqrt{3} ; f(1)=4 ; f(2)=\sqrt{5}$; so the maximum value of the function is 5 and the maximum point is $x=-2$ ". Two students plotted the graph of the 
functions $x \rightarrow \sqrt{x+3}$ and $x \rightarrow 2 \cdot \sqrt{2-x}$ (in the same coordinate system), and thereafter one of them calculated the value $f(-2)$ and wrote this is the maximum value of the function (he made conjectures with the help of the graph), the second one calculated $f(-2)=5$ and $f(-3)=2 \cdot \sqrt{5}$ and she gave the right answer (she also wrote that one of the values $f(-2)$ and $f(-3)$ must be the maximum of the function, because $2 \cdot \sqrt{2-x}$ is more significant than $\sqrt{x+3}) .2$ students wrote that the maximum value is $f(2)=\sqrt{5}$ because $x=2$ is the maximum of the domain. One student thought that the maximum point of the function must be $x=-3$ or $x=2$ (the marginal points of the domain) so the maximum value is $f(-3)=2 \cdot \sqrt{5}$. One student solved the equation $\sqrt{x+3}=2 \cdot \sqrt{2-x}$ and he concluded that the maximum point is $x=1$ and the maximum value is $f(1)=4$. Other student thought that the maximum point of the function is the solution of the equation $\sqrt{x+3}+2 \cdot \sqrt{2-x}=0$ and she also made mistakes in the equation solving procedure. One student tried to find the maximum of the function $f^{2}(x)$ (she obtained $f^{2}(x)=11-3 \cdot x+4 \cdot \sqrt{(x+3) \cdot(2-x)}$ ), other student found the function $f^{4}(x)$ (by a double squaring), but both of them did not know how to handle these complicated expressions. One student stated $f^{2}(x)=x+3+4 \cdot(2-x) \Rightarrow f^{2}(x)=-3 \cdot x+11$ (wrong squaring) and he determined the maximum value of $f^{2}(x)=-3 \cdot x+11$ on the domain $x \in[-3,2]$, namely $f^{2}(-3)=20$, and he got the maximum value $f_{\max }=f(-3)=\sqrt{20}$.

Exercise 3 (grades 10 and 11): Find the maximum of the product $a \cdot b$ where $a+2 \cdot b=4$ and $a, b \geq 0$ !

Table 3

\begin{tabular}{|c|c|c|}
\hline & 10th grade & 11th grade \\
\hline Right answer & 20 & 12 \\
\hline Wrong answer & 20 & 17 \\
\hline No response & 6 & 4 \\
\hline
\end{tabular}

5 grade 10 students and 2 grade 11 students considered the product $a \cdot b$ as a quadratic function $f(b)=-2 \cdot(b-1)^{2}+2$, the maximum point of the function is at $b=1$ and the maximum value of the function is $f(1)=2$ (one of our expected answers). One grade 11 student stated that $\frac{a+2 \cdot b}{2} \geq \sqrt{a \cdot 2 \cdot b} \quad \forall a, b>0$ (and the equality $\frac{a+2 \cdot b}{2}=\sqrt{a \cdot 2 \cdot b}$ holds if and only if $a=2 \cdot b$ ), he solved the system of equations $a+2 \cdot b=4 ; a \cdot 2 \cdot b=4$ and finally he gave the right answer. Other grade 11 student considered the system of equations $a \cdot b=x ; a+2 \cdot b=4$ and she get to the quadratic equation $-2 \cdot b^{2}+4 \cdot b-x=0$; she stated the value of 
$x$ becomes a maximum when the discriminant of the equation is equal to 0 , so $16-8 \cdot x=0$ and $x=2$ follows. We have to mention that the proper and complete reasoning is: if $b$ is a positive real number then the discriminant of the equation must be a non-negative number so $16-8 \cdot x \geq 0$ and $x \leq 2$ follows, therefore the maximum value of $x$ is 2.7 grade 10 students and 5 grade 11 students gave certain values to the variable elements $a$ and $b$ (values which satisfy the $a+2 \cdot b=4$ condition) then they analized the values of the product $a \cdot b$ in order to find out its maximum value (all of them gave the right answer), for example here is one grade 10 student's solution: " $a=1, b=1,5 \Rightarrow a \cdot b=1,5 ; a=2, b=1 \Rightarrow a \cdot b=2$; $a=3, b=0,5 \Rightarrow a \cdot b=1,5 ; a=3,2, b=0,4 \Rightarrow a \cdot b=1,28 ; a=3,8, b=$ $0,1 \Rightarrow a \cdot b=0,38$; so we can see that the product is a maximum when $a=2$ and $b=1$ and the maximum value is $a \cdot b=2^{\prime \prime} .8$ grade 10 students and 3 grade 11 students gave the right answer without any argumentation, or with a weak reasoning like this (one grade 10 student argued in this way): "if the product is a maximum both of $a$ and $b$ must be no less then 1 , so $a=2, b=1$ and the maximum of the product is $a \cdot b=2 " .6$ grade 10 students obtained $a=b=\frac{4}{3}$ and $a \cdot b=\frac{16}{9}$ making the following typical mistake: $a \cdot b$ becomes a maximum when $a=b$ and, from the condition $a+2 \cdot b=4, a=b=\frac{4}{3}$ follows (two of them explained that the equality $\sqrt{a \cdot b}=\frac{a+b}{2}$ holds if and only if $a=b$ ). The same result was obtained by 4 grade 10 students in the following way (we trace one student's work): "the product becomes a maximum if the equality $\sqrt{a \cdot b}=\frac{a+b}{2}$ holds, so $\sqrt{(4-2 \cdot b) \cdot b}=\frac{4-2 \cdot b+b}{2}$ follows; then (after some calculations) we get $9 \cdot b^{2}-24 \cdot b+16=0$ and $b=\frac{4}{3}$ follows" (although they have failed we can see that grade 10 students know more about the inequality between means than the grade 11 ones). Almost in the same way one grade 11 student solved succesfully the system of equations $\sqrt{a \cdot b}=\frac{a+b}{2}$ and $a+2 \cdot b=4$. One grade 10 student's interesting approach was: we have to divide number 4 in three equal parts, so $4: 3=1, \dot{3}$, therefore $a=1, \dot{3}$ and $b=2, \dot{6}$ (he omits to check as well) and the maximum of the product is $a \cdot b=1, \dot{6} \cdot 2, \dot{6}=3, \dot{5}$. One grade 11 student found out that this problem is analogous with the following one: we wish to maximize the area of a pen, $a$ is the length of the pen, $b$ is the width of the pen, and the total amount of fencing is given to be $a+2 \cdot b=4$, because the pen is situated near a river (and we do not build any fencing on this side); but this student made the mistake $a=b$ (as others did) and he found $A_{\max }=\frac{16}{9}$. Many students realized that they have to find the maximum value of the $f(b)=2 \cdot b \cdot(-b+2)$ function, but they did not know how to continue, although this is a simple quadratic function. 


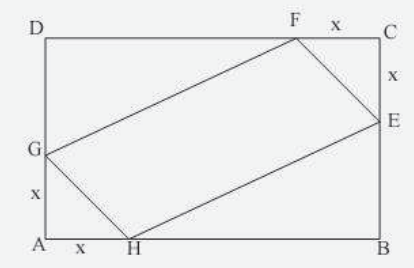

Figure 3

Exercise 4 (grades 10 and 11): The length of the sides of a rectangle $A B C D$ are $A B=10 \mathrm{~cm}$ and $B C=6 \mathrm{~cm}$, respectively. On the sides of the rectangle we consider the points $E, F, G$ and $H$ such that $C E=C F=A H=A G=x$ (see Figure 3). Find the maximum of the area of the parallelogram $E F G H$ !

Our expected answers were the following:

Pattern 1: The area of the parallelogram is $A=60-x^{2}-(10-x) \cdot(6-x)=$ $2 \cdot x \cdot(-x+8)$. This quadratic function has the maximum point $x=4$ and its maximum value is $f(4)=32$.

Pattern 2: We use the inequality between geometric and arithmetic means:

$$
\sqrt{x \cdot(-x+8)} \leq \frac{x+(-x+8)}{2}=4
$$

so $A \leq 32$ and $A_{\max }=32$ follows.

Pattern 3: The sum $x+(-x+8)=8$ is given so we can apply Theorem 3 from Section 2.1. Therefore the product $x \cdot(-x+8)$ becomes a maximum when $x=-x+8$ and so $x=4$ follows.

Table 4

\begin{tabular}{|c|c|c|}
\hline & 10th grade & 11th grade \\
\hline Right answer & 6 & 1 \\
\hline Wrong answer & 32 & 16 \\
\hline No response & 8 & 16 \\
\hline
\end{tabular}

As Table 4 shows the grade 10 students were more succesfull than the grade 11 ones. 6 grade 10 students used Pattern 1 and solved the problem properly. The rest of the students omited this problem or gave wrong answers. One student 
wrote: the area of the parallelogram is $A=60-x^{2}-(10-x) \cdot(6-x)$ and this area becomes a maximum when $x^{2}+(10-x) \cdot(6-x)$ becomes a minimum (and he did not know how to continue). Another student wrote $A=60-x^{2}-(10-x)^{2}$ (she considered all of the triangles are isosceles) thereafter she made the calculations properly, but she gave a wrong answer due to the wrong formula. 2 students thought that the area becomes a maximum when the length of the side $E F$ of the parallelogram becomes a maximum so $x=6.2$ students wrote that $F$ must be the midpoint of the segment $C D$ if the area of the parallelogram is a maximum; 3 students considered that $E$ must be the midpoint of the segment $B C$ when the maximum of the area is attained. 3 students thought the area is a maximum when the parallelogram becomes a rhombus, so $G H=G F$ and they solved the equation $x \cdot \sqrt{2}=\sqrt{(10-x)^{2}+(6-x)^{2}}$ and they got $x=4,25$. Other student argued: "the area becomes a maximum when the parallelogram becomes a rectangle and of all rectangles the square has the maximum area"; he also solved the equation $x \cdot \sqrt{2}=\sqrt{2 \cdot x^{2}-32 \cdot x+136}$ and he got $x=4,25$ (this student knows some rules concerning the extreme value problems, but he does not know how to apply this knowledge and he did not check the validity of his statements). Other student's interesting, but lacunary argument: if $F$ is the midpoint of the segment $C D$ then $x=5$; if $E$ is the midpoint of the segment $B C$ then $x=3$; the area becomes a maximum when $x$ is equal to the arithmetic mean of these two values so $x=\frac{5+3}{2}=4$. One student considered certain $x$ values and calculated the corresponding values of the area: " $x=2 \mathrm{~cm} \rightarrow A=24 \mathrm{~cm}^{2}$; $x=3 \mathrm{~cm} \rightarrow A=28 \mathrm{~cm}^{2} ; x=4 \mathrm{~cm} \rightarrow A=32 \mathrm{~cm}^{2}$ " and she conclude "the area becomes a maximum when $x=4 \mathrm{~cm}$ and the maximum value is $A_{\text {max }}=32 \mathrm{~cm}^{2}$ " . Another student argued in the following way: " $a \cdot b=\max$ if $a=b$, this means the parallelogram becomes a square; it is not possible; so $\sqrt{a \cdot b} \leq \frac{a+b}{2} \Rightarrow$ $\sqrt{a \cdot b} \leq \frac{\sqrt{2 \cdot x^{2}}+\sqrt{2 \cdot x^{2}-32 \cdot x+136}}{2} "$; at this step she abandoned.

The grade 11 students' works were weaker than those of the grade 10 students. Only one grade 11 student gave the right answer, his solution is the following: $A=60-x^{2}-(6-x) \cdot(10-x)=-2 \cdot x^{2}+16 \cdot x=-2 \cdot(x-4)^{2}+32$, then he sketched the graph of the function $f(x)=-2 \cdot(x-4)^{2}+32$ and gave $x=4$ and $A_{\max }=32$. One student wrote $A=60-2 \cdot x^{2}-2 \cdot(6-x) \cdot(10-x)=-4 \cdot x^{2}+32 \cdot x-60$ (wrong formula); then he solved the equation $-4 \cdot x^{2}+32 \cdot x-60=0$ and got $x_{1}=3$ and $x_{2}=5$ ; then $x=\frac{5+3}{2}=4$ and $A_{\max }=4$ followed. One student wrote that the area becomes a maximum when $x$ is as small as possible, so $x=0$. Other student gave the value of $x$ in a strange way: $A=(10-2 \cdot x) \cdot(6-2 \cdot x)=60 \Rightarrow 4 \cdot x^{2}-32 \cdot x=0$; the roots of the equation are $x_{1}=0$ and $x_{2}=8$ and the area becomes a maximum 
when $x=\frac{0+8}{2}=4.5$ students got the formula $A=-2 \cdot x^{2}+16 \cdot x$ but they did not know how to continue. The rest of the students just started the work (they made a picture or wrote one or two formulas) and they gave up, so it is not worth to present their works.

\section{Summary and Conclusions}

Based on the evaluation of the answers we have come to some conclusions. The maximum and minimum problems are difficult enough for secondary school students, this fact is seen in the large number of wrong answers. Also, we can see that the grade 10 students' results were slightly better than the results of the grade 11 ones. Besides efficienty, the grade 10 students apply more adequate methods than the grade 11 ones to solve the extreme value problems. In our opinion, this is due to the fact that the curriculum for grade 11 students does not prescribe any kind of maximum-minimum problem solving, these kind of exercises were not practiced for over a year.

A large number of students think that an extreme value problem somehow involves the equality of two quantities, so they search for two quantities which must be equal and they often give erroneous results. The main cause of these errors is the misinterpretation of the basic inequalities between arithmetic, geometric and square means. We can also say that the students textbooks mostly deal with extremum problems where the equality between two or more quantities delivers the right answer (see [1] and [3]). To make an improvement in this sense we consider it is necessary to deal with problems like Exercise 3 and 4 from Section 3, in the secondary school educational processes. Many students gave the right answer after they calculated the value of a function or expression for several certain values of the variable. This is an eloquent proof that most of them did not find any way to solve the problem and they ultimately appealed to this lacunary method. The students face difficulties when they have to synthetize knowledge concerning functions, algebraic expressions or geometry. It is also difficult for them to find analogous problems to the just discussed problem.

In our opinion a serious improvement is necessary in the extreme value problem solving activities. It is necessary to inlarge the number of methods which are adequate to solve extreme value problems (we have shown some of them in Section 2, others can be found in [2], [5] and [6]). It is also necessary to deal with a greater variety of problems, not only those focused on the inequalities between means. And, after all, the extrem value problems have their rightful place in 
almost every chapter of the secondary school Mathematics education, not only in the grade 10 curriculum.

\section{References}

[1] L. Gerőcs, Gy. Orosz, J. Paróczay and J. Szászné Simon, Matematika-Gyakorló és érettségire felkészÍtő feladatgyüjtemény I., Nemzeti Tankönyvkiadó, Budapest, 2008.

[2] E. Hódi, Szélsőérték-feladatok elemi megoldása, Typotex, Budapest, 1994.

[3] J. Kosztolányi, I. Kovács, K. Pintér, J. Urbán and I. Vincze, SokszÍnü matematika, Mozaik, Szeged, 2003.

[4] C. Nastasescu, Exercitii si probleme de algebra clasele IX-XII, Editura Didactica si Pedagogica, Bucuresti, 1992.

[5] C. P. Nicolescu, Sinteze de matematica, Editura Albatros, Bucuresti, 1990.

[6] G. Pólya, Mathematics and Plausible Reasoning Vol. I., Induction and Analogy in Mathematics, Princeton University Press, Princeton, 1968.

[7] http://kerettanterv.ofi.hu/03_melleklet_9-12/index_4_gimn.html.

ZSOLT FÜLÖP

BOLYAI INSTITUTE

UNIVERSITY OF SZEGED

HUNGARY

E-mail: fulop.zs32@freemail.hu

(Received October, 2014) 